\title{
Perioperative Care Considerations and Management of Complications Involving Surgical and Technology Procedures
}

\author{
Richard D. Gentile, MD, MBA ${ }^{1}$ \\ ${ }^{1}$ Facial Plastic and Aesthetic Laser Center, Youngstown, Ohio \\ Facial Plast Surg 2018;34:579-587.
}

\begin{abstract}
Address for correspondence Richard D. Gentile, MD, MBA, Facial Plastic and Aesthetic Laser Center - Medical Director, 6505 Market A103, Youngstown 44512, Ohio (e-mail: dr-gentile@msn.com).
\end{abstract}

\author{
Abstract \\ Keywords \\ - technology \\ - facial plastic surgery \\ - burn \\ - sequelae \\ - complications
}

Technology impacts the lives of everyone nowadays and has had perhaps a greater impact on the lives of facial plastic surgeons and the patients they seek to help with both surgery and procedures. This article will discuss modern technology's impact on intervening in the aging process including technology used for facial and skin rejuvenation and reconstruction concentrating on the techniques and care to minimize complications as well as dealing with complication from the treatments.
The perioperative care considerations in facial plastic surgery include planning and assessment of all potential operative concerns that may create problems for the patient in the immediate and later postoperative course until all recovery and healing has occurred. It also involves a strong commitment to patient education during the process. It is often stated that the best way to manage complications is to prevent them from occurring, so patient assessment is very important but perhaps even more important is the development of a productive patient-doctor relationship that promotes not only effective communication and understanding but also provides a good milieu for patient education so that the treatment objectives are understood and that patients will respond to their postoperative instruction in such a way that their recovery is expedited after procedures. To achieve these goals, the surgeon and members of the surgeon's team need to dedicate adequate resources particularly time in educating the patient about the anticipated objectives and thoroughly prepare the patient for the upcoming procedures. Realistic information about the healing process, bruising, swelling, and potential complications needs to be discussed at length with the patient so that no surprises or misunderstandings exist.

\section{Perioperative Care Considerations in Technology-Based Procedures}

The reference to "technology-based procedures" usually refers to the use of energy-based devices such as lasers, light, radio- frequency, ultrasound, or plasma devices. We will discuss some treatment considerations that will help to increase patient comfort and reduce sequelae or complications. Prior to treating patients with ablative resurfacing, an antiviral oral regimen may be prescribed. Valacyclovir and famciclovir have better absorption and better bioavailability than acyclovir. Topical anesthetic may be applied to the skin before the procedure to reduce pain. During and immediately after the procedure, cooling the skin with air or ice packs may reduce pain. To avoid postinflammatory hyperpigmentation (PIH) after treatment, patients should avoid direct sun exposure and use a broadspectrum sunscreen for several weeks. A skin-lightening cream may also be prescribed. The treated lesions, such as freckles and lentigines, will generally become darker and occasionally crusted approximately 3 days after the treatment. It gives a "dirty look" which will persist for 3 to 5 days after treatment and slough off thereafter. Regular (daily) gentle cleansing (e.g., with normal saline) and applying moisturizers (e.g., aqueous cream) two to three times a day will help hasten clearance of the crust. Slight redness may occur after the laser and light procedures, but usually disappears spontaneously after a few hours. Uncommon side effects include superficial blistering, especially when treating tanned or darker skin tones. This can be prevented by avoiding treating patients with tanned skin. Properly chosen wavelengths of light used with appropriate pulse durations can selectively alter pigmented cells and disrupt exogenous and endogenous pigment in a manner that leaves the adjacent skin intact and allows for the effective and
Issue Theme Postoperative Care in Facial Plastic Surgery; Guest Editor: Alwyn D'Souza, MBBS, FRCS Eng, FRCS (ORL-HNS), PGCertMedEd, EBFPRS
Copyright (c) 2018 by Thieme Medical Publishers, Inc., 333 Seventh Avenue, New York, NY 10001, USA. Tel: +1(212) 584-4662. 
safe treatment of many cosmetic skin pigmentary disorders which are common in aging skin. Risk of PIH can be reduced with proper patient selection, appropriate laser/light device selection, and good perioperative skin care.

\section{Postsurgical Care and Management of Complications Facial Plastic Surgery and Technology-Based Procedures}

The effort to rejuvenate the aging neck and face can have intermediate consequences before the desired result is achieved. These consequences, as described by Truswell, ${ }^{1}$ can be either unavoidable side effects of surgery and aesthetic procedures, such as bruising or swelling, or can take the form of incisional scarring and complications. In contrast to sequelae, which are usually expected with aesthetic surgery and aesthetic procedures of the head and neck, complications of such surgery and aesthetic procedures are not inevitable. It is important that the aesthetic surgeon distinguish the several types of sequelae and side effects of surgery and aesthetic procedures from the less frequent complications that may occur. Complications can result from poor surgical planning or poor surgical execution(-Fig.1), or more commonly, from undetermined circumstances that are difficult to predict. We will discuss the complications after aesthetic surgery for rejuvenation of the face and neck.

\section{Problems in Wound Recovery}

\section{Hematomas and Seromas}

Postoperative fluid collections, most commonly in the form of hematomas from bleeding or the oozing of blood, can be seen in as many as $15 \%$ of patients undergoing face/neck lift surgery. ${ }^{2}$ In properly performed cosmetic surgery of the neck, small pockets of serous fluid and blood can infiltrate the dermis, subcutaneous plane, and underlying muscle structure, resulting in the perception of "lumps and bumps." These small collections of fluid are normal in the initial postoperative period, and by definition cannot be aspirated. Supportive garments, massage, and time will allow their complete resolution. These small collections of fluid differ from a localized collection of blood, which is defined as a hematoma. If detected at an early point, within hours after surgery, a hematoma can be evacuated before causing the separation of skin flaps in the neck from the underlying muscle structure. This can be accomplished by needle aspiration if minimal incisions were used, or more commonly by opening the postauricular or submental incision, placing or replacing the subcutaneous drains, and applying a pressure dressing. If a longer period passes before the treatment of a hematoma, the subcutaneous blood will have coagulated and cannot be aspirated. Immediate treatment is necessary for larger hematomas or those that are actively bleeding or expanding, because the separated skin flap in such cases is exposed to the acidic effects of degenerating blood products that put the flap at risk of necrosis and permanent tissue loss. This can be done by reopening the postauricular or submental incision and can be accomplished in an office-based or ambulatory surgical facility. Patients may have to stay overnight for observation during the 12 hours after treatment. If the hematoma is relatively small, it can usually be successfully treated with observation and repeated needle aspiration, although aspiration usually cannot be accomplished until the hematoma begins to liquefy, which typically occurs on postoperative
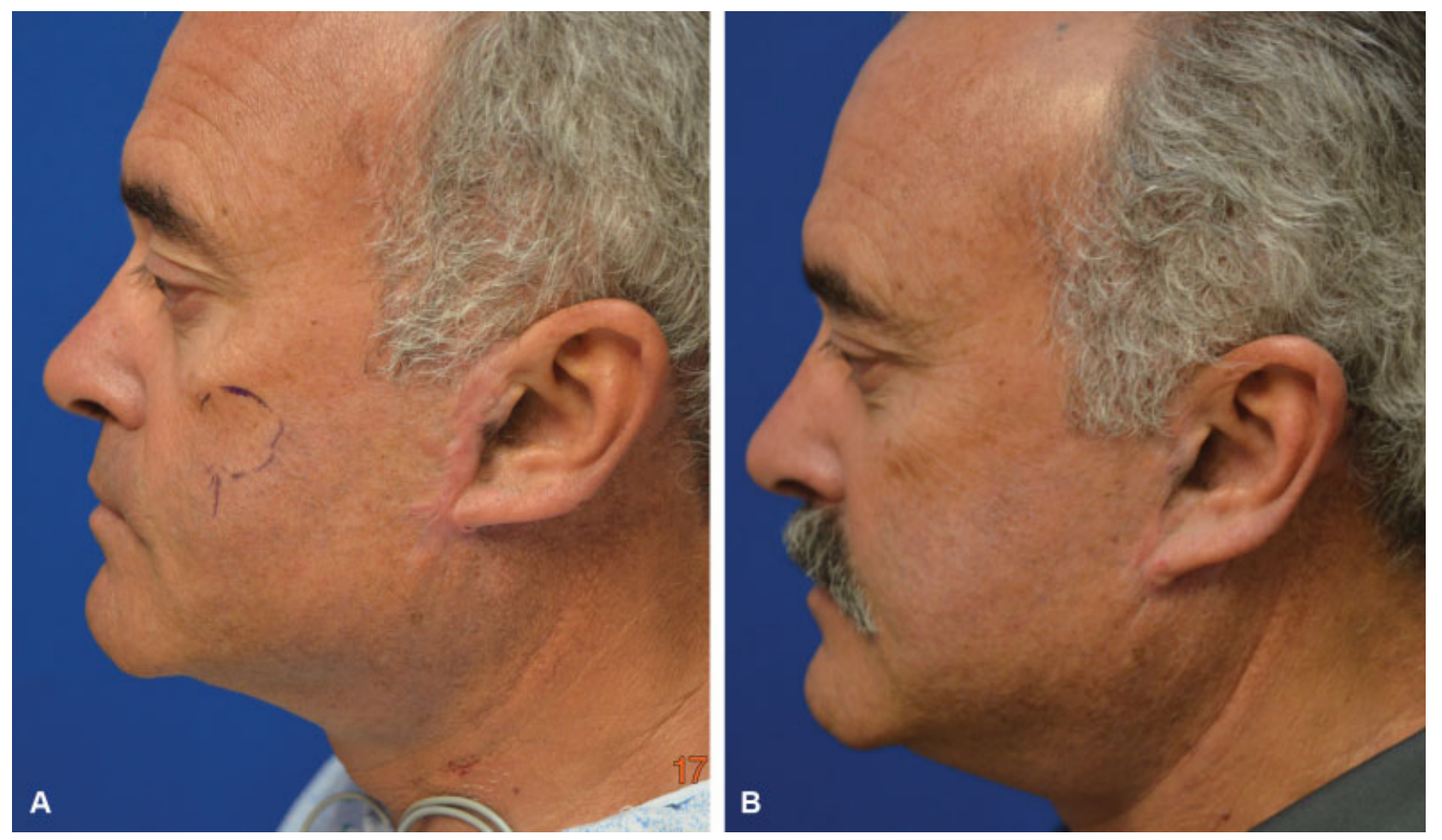

Fig. 1 (A) Excessive skin tension on the facial flap can result in tension created by hypertrophic scarring in the preauricular region. (B) Correction of tension associated with hypertrophic scarring can be improved with extensive undermining and skin recruitment. This patient has wide undermining with fiber laser. 
days 8 to 12 . Delayed healing and hyperpigmentation are among other possible complications of rejuvenation surgery of the head or neck. A seroma exists when localized fluid is present in the subcutaneous plane. Often the result of an untreated hematoma, a seroma can also separate a skin flap from the underlying muscle and cause irregularities in contour, such as skin rippling. As in the case of a hematoma, repeated aspiration is the preferred treatment for a seroma. In cases of significant flap separation, a pressure dressing must be applied to avoid the recurrence of a seroma and propagation of the cycle of fluid accumulation, flap separation, and regeneration of seromas.

\section{Infection}

An infection can result from any surgical procedure but is uncommon in facial procedures because of the copious blood supply of the face and neck. Some treatments of the neck, and specifically contouring of fat, will make infections more likely than would the same treatments in the adjacent areas. Necks that require significant fat contouring are more likely to become infected because of inadequate evacuation of denuded fat cells and blood. The fat cells die and mix with blood to become an ideal medium for bacterial growth. Infections of this type usually manifest at about a week postoperatively and present early in the form of isolated painful swelling of the neck. Within days, the infected area becomes erythematous and fluctuant as swelling and pain increase. In most cases, organisms such as Streptococcus or Staphylococcus are the causative agents of such infections, and specific antibiotic coverage for these organisms should be provided. Because organisms on the skin are the usual pathogens in such infections, and most nonpenicillin or cephalosporin antibiotics provide only limited coverage of the spectrum of organisms on the skin, cervical infections of the type described here are more common in penicillinallergic patients. Prompt attention is necessary both to treating cervical infections of the type described above and preventing soft tissue damage from them. Local treatment is also necessary and includes warm compresses and repeated needle aspirations. Because these infections behave like abscesses, their abscess-like acidic environment can cause soft-tissue necrosis, especially of the surrounding subcutaneous fat. This can produce a permanent contour defect from the asymmetric loss of fat. Immediate culture of fluid aspirated from such an infection can improve the efficacy of their antibiotic coverage. If the infection is proximal to a submental or postauricular incision, evacuation can be done through these incisions. If the incision is not proximal and frequent follow-up care is needed, localized aspiration can be used to treat the infection without disturbing an incision that is healing well. Today, any infection that does not respond appropriately to the treatment described above should be considered as possibly being caused by methicillin-resistant Staphylococcus aureus (MRSA). This organism is an increasing problem, and its antibiotic resistance must be addressed in a timely fashion. Hospital-based procedures, older age of a patient, or recent housing of a patient in institutions such as hospitals or nursing homes are risk factors for MRSA infection. Patients with these infections often require intravenous antibiotic treatment. Persistent, indolent, or recurrent granulomas, especially at the sites of incisions, can be caused by atypical mycobacteria, which are revealed by microbiological culture.

\section{Sialoma}

The occurrence of sialoma or salivary drainage from the submandibular gland is very unusual in most uncomplicated surgical procedures on the neck. Procedures associated with partial excision of a submandibular gland can be complicated by sialoma, but this is again very infrequent. A parotid fistula can lead to the accumulation of saliva in the neck in patients undergoing rhytidectomy, but this complication too is rare. Accumulation of fluid that does not respond to simple treatment methods should be suspected as possibly being a sialoma. Current treatment of these lesions now includes Botox to block the innervation of the affected gland.

\section{Complications Involving the Skin and Dermal Appendages}

\section{Skin Injury and Necrosis}

Because cosmetic surgery of the neck usually requires the separation of skin of the neck from the underlying platysma muscle, the resultant skin flap can be vulnerable to ischemic complications. The incidence of skin necrosis in cervical and facial surgery is reported to range from 3 to $5 \%$, with the most frequently involved areas being those of a postauricular incision and flap, followed by sites of flap closure adjacent to the superior auricle and hair tuft, and of flaps adjacent to the lobule of the ear. When an area of the skin on the neck becomes ischemic, it can break down, with ensuing tissue loss and permanent visible scarring. Etiologies of skin breakdown vary, and most commonly include overaggressive liposuction of the fat of a raised skin flap or aggressive cautery of bleeding vessels on the undersurface of a skin flap. In these situations, the skin breakdown can be delayed and show only the minimal initial sign of sloughed epithelium as an indicator of transmural ischemic skin injury. The injury and tissue loss in such cases are usually small, but can be cosmetically obvious, and also because the ischemia are slow to heal. Denuding the ischemic edges of such a site and suturing the healthy tissue edges can expedite the healing process. Other causes of skin injury and tissue loss are untreated hematomas or infections, and distressed healing in cigarette smokers. In these situations, the acidic components of both a hematoma and the infectious process, together with skin flap separation, can create softtissue ischemia and, especially if left untreated, lead to necrosis of a skin flap. Cigarette smoking and a history of past cigarette smoking are probably the single greatest factors predisposing to skin necrosis after surgery of the neck and face, with the risk of flap necrosis for smokers who continue to smoke up to the time of their surgery and postoperatively being 12.5 times that for nonsmokers. ${ }^{2}$ Diabetic patients are also at risk of problematic healing after surgery of the face and neck, but not to the same extent as cigarette smokers. Tight compression dressings can contribute to vascular compromise 

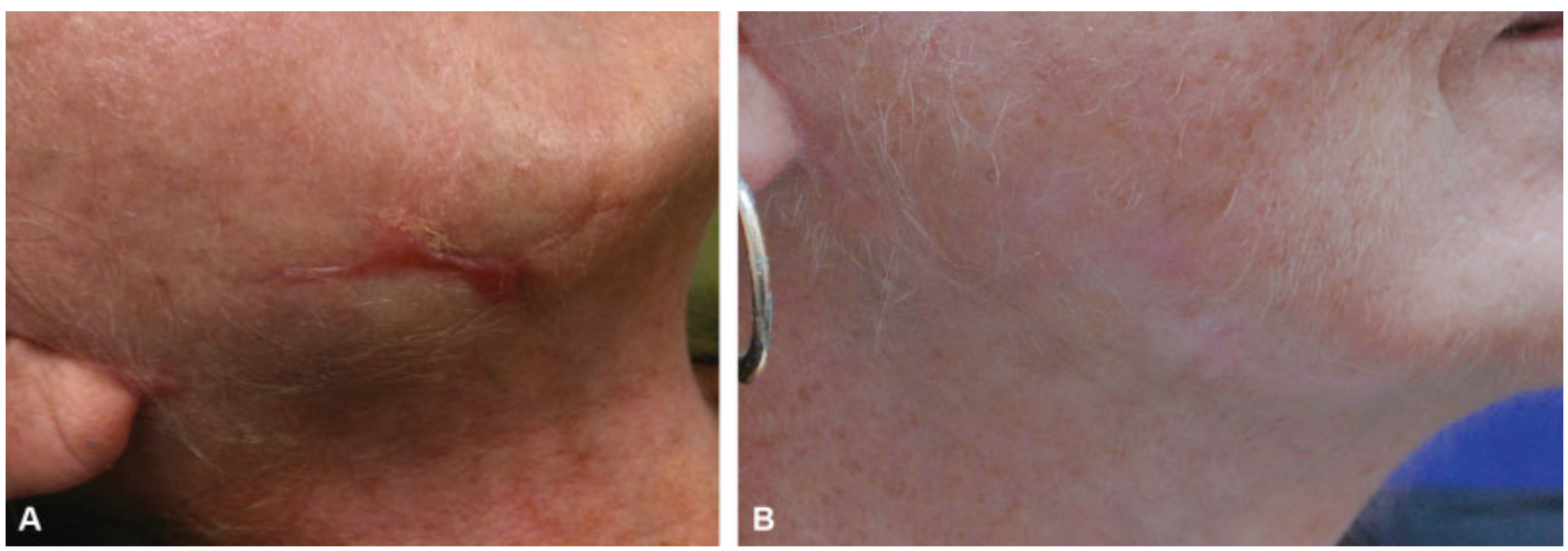

Fig. 2 (A) Skin necrosis and eventual healing can result in noticeable scarring. (B) Noticeable scarring reduced with triamcinolone injections, pulsed light, and fractional $\mathrm{CO}_{2}$ laser treatments.

in a flap, and the need for such dressings should address their potential threat to wound healing. In patients with delayed healing or skin necrosis of a flap, the underlying causative process should be permitted to resolve completely so that secondary repair of the flap can proceed if necessary. Some areas will heal satisfactorily without secondary intervention. Treatment consists of conservative wound care and debridement, followed by laser treatment or scar revision of any full-thickness wounds after the eschar has resolved. The use of fractional ablative lasers as well as pulsed dye lasers has been useful for blending some epidermal irregularities into the skin contour in patients with delayed healing or skin necrosis and is also helpful for irregular surgical scars (-Fig. 2).

\section{Changes in Skin Pigment or Texture}

Excessive tension on the skin can lead to the lightening of the adjacent skin in patients undergoing neck lifts, and persons with Fitzpatrick types IV to VI skin can develop postinflammatory hyperpigmentation after such surgery (-Fig. 3). Patients should avoid tanning after undergoing face or neck lifts. Patients who have telangiectatic vessels may experience a postoperative increase in these lesions, which can be treated with various vascular lasers or intense pulsed light devices.

\section{Redundant Skin}

In aesthetic techniques that remove underlying fat or tighten underlying muscle in the neck without removing excess skin, redundant skin can fold or pleat, creating a defect in contour. In patients with good skin elasticity, the skin will often contour with the underlying structures in the neck and postoperative folds in the skin will resolve. However, these folds may remain if the redundant skin is significant or inelastic. This can be treated either with direct excision of the folded skin or with periauricular incisions and the redraping of a skin flap, with the excision of redundant skin from the flap. The most common location for redundant skinfolds or pleats is the submental region, followed by the earlobe and lateral neck (-Fig. 4).

\section{Hypertrophic Scarring}

Hypertrophic scarring or keloid formation can occur after face and neck lift procedures (-Fig. 5). This commonly occurs behind the ear but can also develop at the site of a posterior hairline incision. A careful history can help identify patients who may be predisposed to this type of abnormal wound healing so that they can be given preoperative counseling. The treatment for hypertrophic scarring is usually triamcinolone (10 or $40 \mathrm{mg}$ ) given intralesionally, or treatment with one of the newer fractional or pulsed dye lasers, which are useful in reducing the bulk of abnormal scar tissue (-Fig. 1). There is evidence that 5 -FU can be used alone or in conjunction with intralesional steroid injections for reducing postprocedure scar tissue. Topical silicone ointments or patches have also been demonstrated to provide improvement in postprocedure scarring.
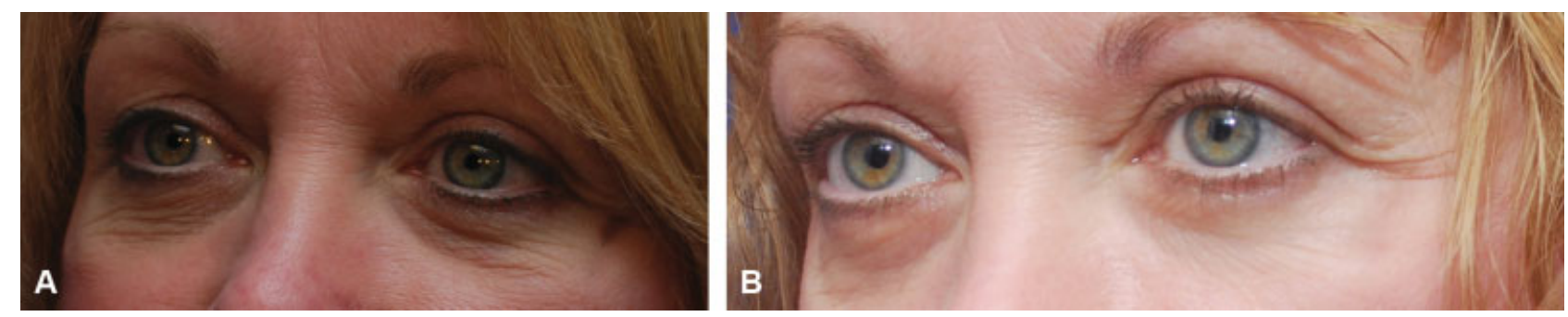

Fig. 3 (A) Hyperpigmentation can result from thermal injury to the facial or neck skin. (B) Hyperpigmentation responds to skin lightening creams and appropriate pigment reducing lasers and light devices. 


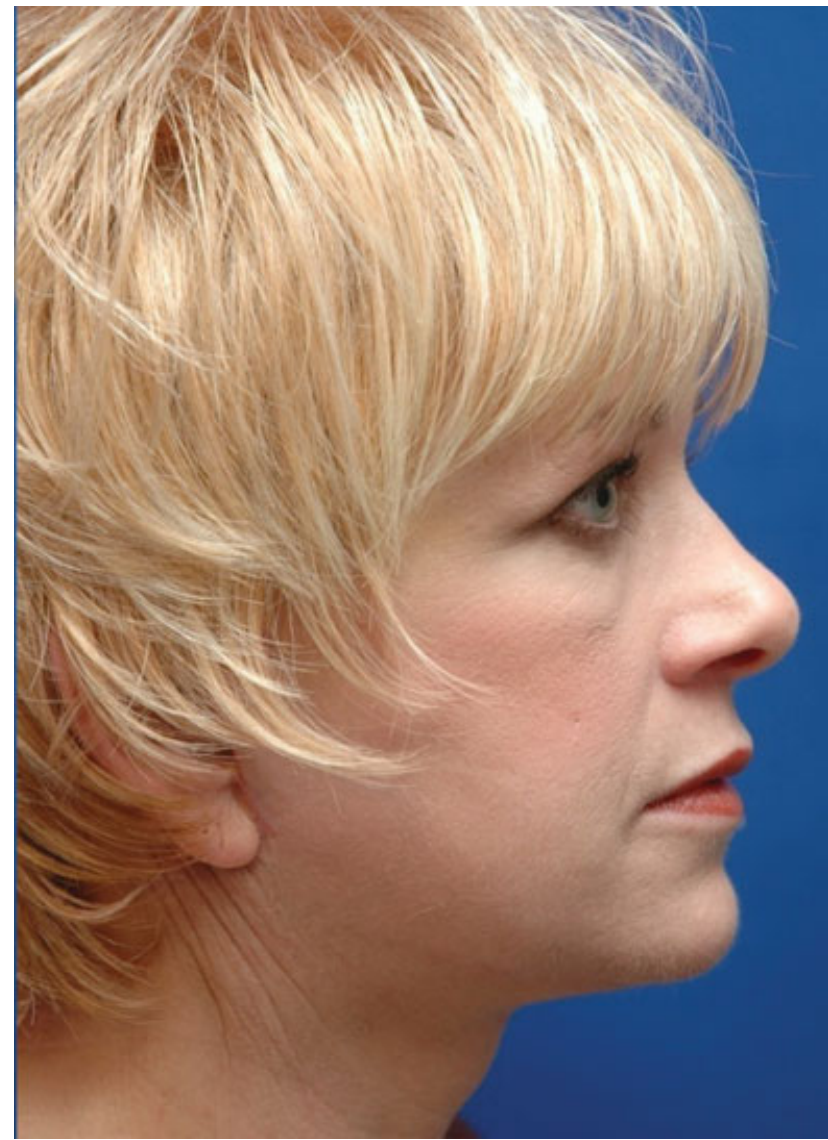

Fig. 4 Lateral neck skin pleating resulted from inadequate length of redraping of the posterior neck skin. Longer incision in the posterior neck can avoid this outcome.

\section{Suture Erosion or Palpability}

Suture erosion through the skin can occur whether absorbable or nonabsorbable sutures are used in aesthetic surgery. If this occurs, the sutures are simply removed. Suture erosion may occur more frequently in patients with thin skin who lack subcutaneous fat. Bulges or bumps can be a problem if a nonabsorbable suture material is used or if knots in a suture are not buried.

\section{Poorly Designed Incisions and Distortions in Anatomy}

Wound closure in rhytidectomy is not only the most time consuming of all the surgical steps in this procedure but is also one of the most important to the patient. Incision planning is important both from the standpoint of technique and because patients expect that their surgery will leave inconspicuous scarring. The surgeon should realize that a significant part of a patient's satisfaction with an aesthetic surgical procedure will rest on the patient's determination of whether any residual scars are conspicuous. Scars must be inconspicuous, and care must also be taken to prevent alterations of the patient's hairline. The preauricular tuft should end up near its original site, and overelevation of the hairline should be avoided. In patients having high temporal incisions, such as those in a classical deep-plane rhytidectomy, the temporal hair tuft must be brought down to counter the vertical elevation of nonhairbearing skin into the region previously occupied by the temporal hair tuft. This can be accomplished by a small flap rotation and the excision of a Burrow's triangle. Lower incisions, at the margin of the hair tuft, will not tend to raise a sideburn, but puckering at the medial extent of the tuft can be a problem with this approach, and the scar from this may be quite visible. By the same token, the posterior incisions in a rhytidectomy should not pull the hairline obliquely backward, and there must be no "step-off" in the postauricular hairline. It is important to control the tension applied to the closure of preauricular incisions to not distort the auricle. Skin excision and tension should be minimal in the region of the lobule to avoid a "pixie," "satyr," or "devil's ear" deformity (-Fig. 6). Careful attention to detail will help avoid the sequelae of poorly healed and distorted incisions.

\section{Hair Loss}

Hair loss can occur infrequently in hair-bearing flaps or near incisions traversing hair-bearing regions of the face and scalp and is thought to occur in 3\% of rhytidectomy procedures. ${ }^{2,3}$
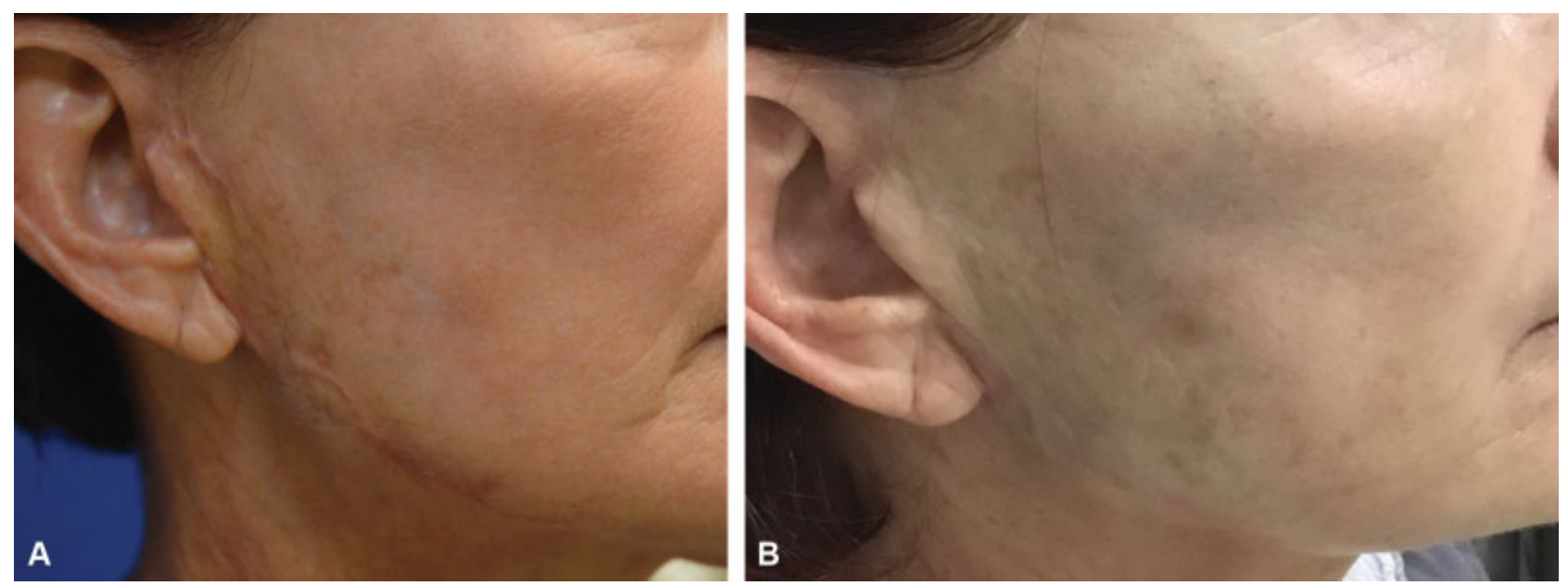

Fig. 5 (A) Hypertrophic scarring can be associated with skin necrosis or thermal injury from lasers and electrosurgical devices. (B) Reduction of hypertrophic scarring by treatment with triamcinolone injections, pulsed light, and fractional $\mathrm{CO}_{2}$ laser treatments. 

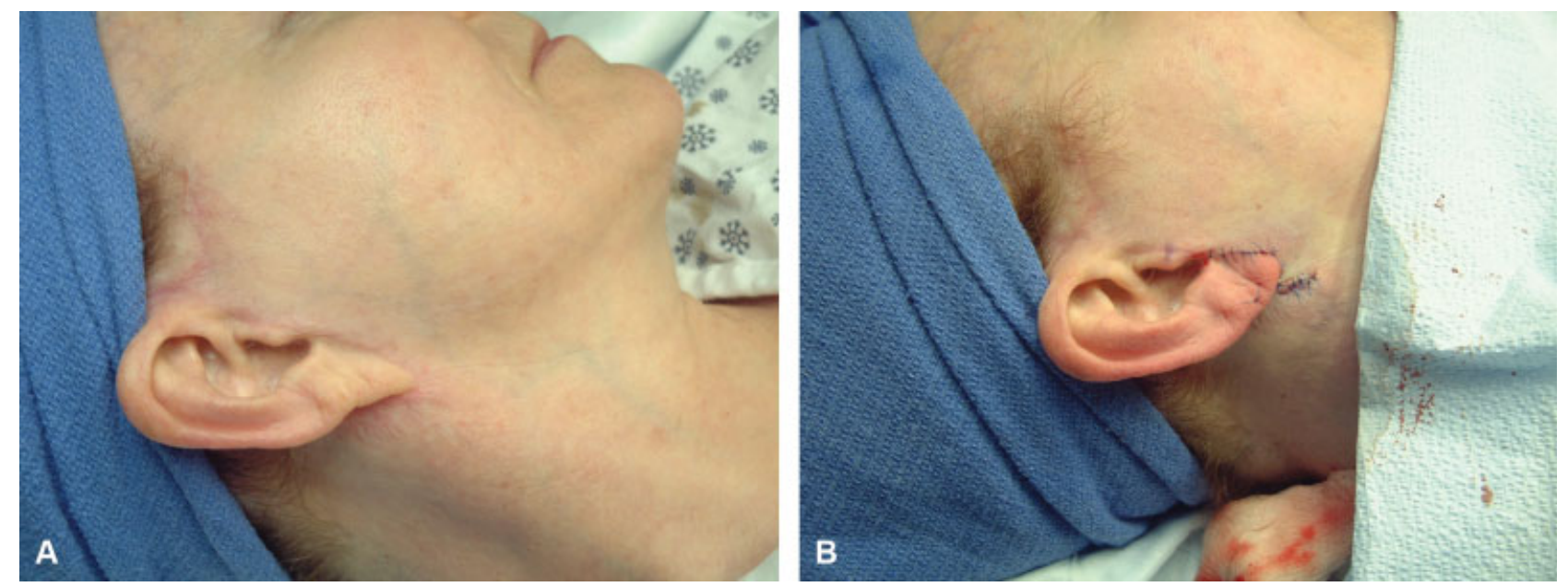

Fig. 6 (A) Pixie ear deformity can be caused by excessive skin excision and tension at the lobule. (B) Improvement in pixie ear deformity with Zplasty.

Although hair loss is unusual after rhytidectomy, it is important to note that factors contributing to it include thinning of hair-bearing flaps, injury from electrosurgery, and excess tension on the skin during wound closure. The prognosis for the regrowth of hair in affected hair-bearing areas is good, and the patient should be reassured until regrowth occurs.

\section{Paradoxical hypertrichosis}

This complication is a relatively common phenomenon in laser hair removal (LHR) and tends to be a problem in individuals with skin types III to V particularly and in the face region. Although the physical consequences of this are minor, it generally tends to cause a lot of distress among its sufferers. The exact mechanism for this "stimulated hair" is unknown, though hormonal influences could play a role and exclusion of an underlying endocrine disorder in females is recommended. The condition is defined as an increase in the color, density, and coarseness of hair following LHR.

\section{Line of Demarcation}

This complication represents a color step-off between treated and nontreated areas and typically occurs in ablative and to a lesser degree in nonablative rejuvenation procedure (-Fig. 7). This may not necessarily be due to high fluences and tends to occur more often in lighter skin types and individuals with actinic bronzing. Measures to minimize this include confining treatments to cosmetic subunits and feathering the edges of the treated areas by using lower fluences and densities, and in skilled hands the use of high repetition mode in a painting fashion.

\section{Nerve Damage}

Although many complications of cervical and facial surgery are temporary, some can be enduring and of much greater concern to the operating surgeon. Nerve damage, whether sensory or motor, can be both temporary and enduring, and temporary sensory deficits are probably among the most common sequelae of surgical procedures on the neck and face, with nearly all patients who undergo such surgery sustaining at least short-term numbness or altered sensation in the face or neck. At the other end of the spectrum is nerve damage that leaves permanent motor or sensory deficits and, aside from serious wound complications that leave permanent scarring and deformity, is among the most problematic complications of face and neck surgery for both the patient and the operating surgeon.

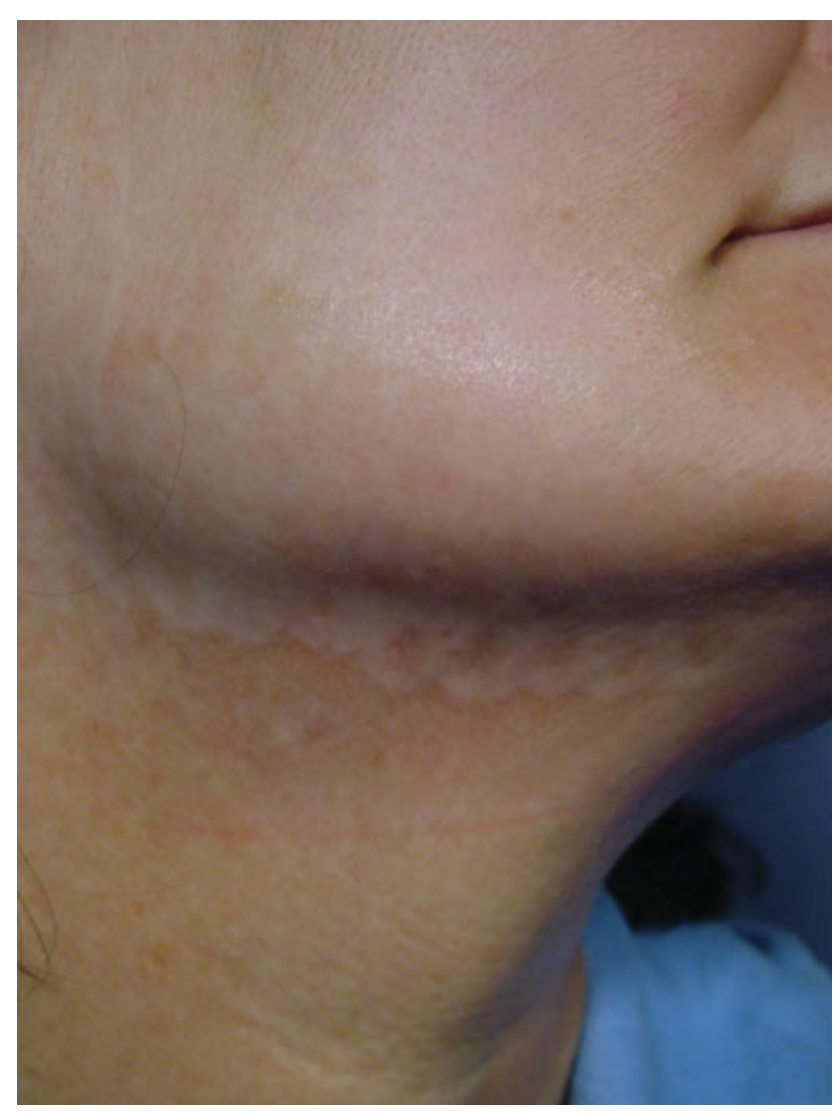

Fig. 7 Line of demarcation/hypopigmentation due to excessive resurfacing energy. Complication can be limited by using energy devices with less thermal destruction 


\section{Greater Auricular Nerve}

Sensory deficits or alterations are a common short-term sequelae of neck or facial surgery and nearly all patients have some degree of sensory deficit following surgery which usually resolves in the weeks or months following surgery. "Numbness" in fact is one of the more troublesome sequelae of surgery and should be discussed with the preoperative patient prior to surgery. The greater auricular nerve or its terminal branches are the most susceptible to being injured in neck or facial surgery with a quoted incidence of up to $7 \%$ of procedures.

\section{Facial Movement Disorders: Marginal Mandibular Nerve Paralysis and Pseudo-Marginal Mandibular Nerve Paralysis}

Temporary or permanent disorders of facial movement can occur in cosmetic surgery of the neck as the result of injury to either a branch of the facial nerve or to the platysma muscle. The marginal mandibular nerve, a branch of the facial nerve, travels in the subplatysmal plane, in the fascia of the submandibular gland, at a breadth of approximately two fingers below the edge of the mandible. Aggressive dissection in the subplatysmal plane can make this nerve vulnerable to injury. Another mechanism of injury to the marginal mandibular nerve occurs in the treatment of a ptotic submandibular gland. Because the nerve lies in the fascia covering the gland, even indirect technique for suspension of the submandibular gland can risk injury to the nerve. Because the nerve innervates the lateral depressor function of the lower lip, an asymmetric smile with a unilateral loss of lateral depressor function is diagnostic of this condition. Apart from an observed direct transection of the nerve, most injuries causing complete loss of function of the marginal mandibular nerve are temporary, and full return of its function typically occurs within 3 months. Incomplete injuries to the nerve can be secondary to edema, and once motion is noted in the depressor function of the lower lip, a complete return of function is very likely. Treatment of complete injuries to the marginal mandibular nerve can include the use of oral steroids, but studies are inconclusive about these drugs' effectiveness in promoting healing of the nerve. Botox injection into the depressor muscle of the contralateral lower lip can mask a deficit in depressor function of the lower lip and is effective in temporarily removing the obvious facial asymmetry that occurs during smiling in this situation. The injection can clearly be repeated indefinitely in cases of permanent injury. Although its gross appearance is like that in a case of nerve injury, paralysis of the structures innervated by the pseudo-marginal mandibular nerve is not a nerve injury but an injury to the platysma muscle. As in the case of nerve injuries, this injury can be either temporary or permanent. Paralysis stemming from injury to the pseudomarginal mandibular nerve is caused by injury to the medial platysma muscle proximal to the mentum. Because the medial or central platysma muscle contributes to the depressor function of the lower lip, injury to the muscle can produce a unilateral loss of depressor function of the lower lip. Diagnosis and differentiation of this condition from injury to the marginal mandibular nerve is done by observing facial movement. In paralysis from injury to the pseudo-marginal mandibular nerve, the loss of depressor function is confined to the medial aspect of the lower lip just lateral to the midline, whereas in marginal mandibular nerve injuries the loss of depressor function is confined to the lateral aspect of the lower lip. The differences in these two effects can be subtle but are clear to the trained observer. In most cases, paralysis from injury to the pseudo-marginal mandibular nerve is temporary and resolves within days to months. It is caused either by edema of the platysma muscle or reversible muscle injury. Steroids have not been shown to be effective in reversing such injury, but Botox can minimize the cosmetic effects of the resultant facial asymmetry in cases of both temporary and permanent paralysis.

\section{Irregularities in Contour}

Irregularities in contour of the neck after rejuvenation surgery usually become more evident as the patient heals postoperative, because early edema after surgery generally obscures these irregularities. The irregularities in contour can culminate in persistent convex deformities, concave deformities, a persistence of platysmal banding, and other abnormalities of contour.

\section{Persistent Convexity}

Persistent convexity or bulges ( - Fig. 8), folds, or ridges in the neck can involve any of its tissue layers, ranging from the skin, fat, and muscle to deeper structures such as the salivary glands. Because the fascia and muscular support of the submandibular gland weaken through the effects of gravity, a cosmetically visible ptotic submandibular gland is common in many necks. Before cosmetic surgery is done, the gland is hidden within a ptotic platysma muscle and soft tissue of the neck. Once the platysma muscle is tightened, fat in the neck is contoured, and skin on the neck is tightened, the untreated ptotic gland becomes evident. Views differ about its treatment because the submandibular gland is deep to the platysma and has the marginal mandibular branch of the facial nerve lying in its fascia. Nonaggressive approaches will not correct a ptotic gland. The patient should be advised that the gland is a normal structure with risks involved in its repositioning. More aggressive techniques for repositioning the submandibular gland can range from suture suspension to resection of the gland. These techniques can cause injury to the marginal mandibular branch of the facial nerve. Besides the submandibular gland, other structures deep to the platysma muscle are also subject to the effects of aging and gravity and can also be undertreated in cosmetic surgery of the neck. A ptotic digastric muscle is often confused with a ptotic platysma muscle, each appearing as banding in the neck. In most cases, a ptotic platysma muscle is medially dehiscent and not attached to the hyoid bone. The digastric muscle is deep to the platysma, more laterally based, and attached to the hyoid bone. Because its medial belly can be approximated when the platysma muscle is imbricated, the digastric muscle is often treated when treating the platysma. 

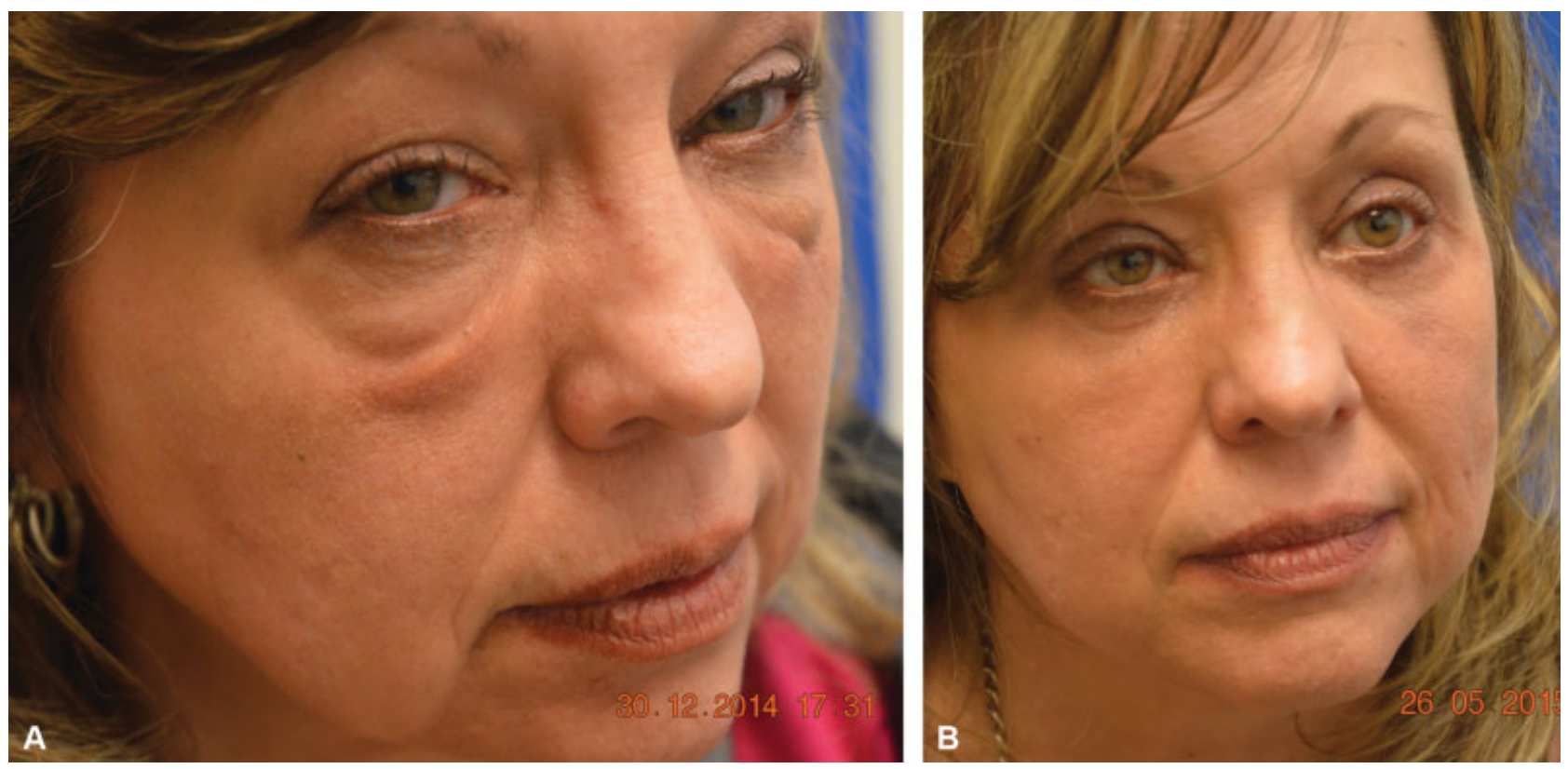

Fig. 8 (A) Convexity due to improperly placed filler injection. (B) Correction with contour lower lid blepharoplasty that corrects lid cheek junction irregularities.

If the platysma is adequately treated, evident persistent lateral banding in the neck is often caused by an untreated ptotic digastric muscle. This is most common in older, very loose necks in which it is difficult to differentiate preoperatively between excessive fat, a ptotic platysma muscle, and a ptotic digastric muscle, and significant improvement of the first two of these conditions leaves the ptotic digastric muscle evident. Treatment for a ptotic digastric muscle is like that for platysmal banding but is done in a deeper plane by medially imbricating the bands of the ptotic digastric muscle. More aggressive techniques involve inferior muscle releases and other muscle-transection techniques but are less common today than formerly because of secondary problems at the muscle transection site. Persistent platysmal banding occurs when the platysma muscle is inadequately imbricated medially during cosmetic surgery of the neck. This most often results when a misdiagnosis calls for lipectomy in the neck when in fact an underlying ptotic platysma muscle is hidden in the soft tissue. This usually occurs with significant aging-related changes in the neck. The platysma muscle can also be undertreated, with persistent platysmal banding as a result. Fat is another component of the aging neck that can be undertreated, creating a defect in contour. Persistent fat in the neck can present as any fullness that would not be of muscular or salivary gland origin. A diagnosis of this should not be made until most of the soft-tissue edema following surgery has resolved, over a period of many weeks to months. Although most common submentally, persistent fat can occur in any area of the neck. It is most common in necks that require significant lipectomy and contouring, resulting from uneven contouring of the fat. A dilute solution of a steroid such as Kenalog can be used in small defined areas to cause fat to dissolve but control is difficult and overcorrection is a possibility. Isolated lipect- omy is usually the treatment of choice. Overly aggressive surgery of the neck can also create a persistent convexity in the neck, in the specific form of banding around the hyoid bone. Because the hyoid bone is an attachment for deeper muscles of the neck such as the digastric muscle, and not the platysma, overimbrication of the platysma inferior to the hyoid bone will create a visible band of muscle over the hyoid bone. This can be more prominent in excessively defatted necks. Releasing an imbrication suture, either through the submental incision made for inserting the suture or directly, can remove this defect in contour.

\section{Persistent Concavity}

A persistent concavity in the neck or depressions or irregularities in its contour can involve any of the layers of tissue in the neck, whether the skin, fat, muscle, or deeper structures. Many problems in this category are created by overly aggressive surgery of the neck. One of the more common defects in contour of the neck is muscle banding. This is caused by overly aggressive defatting. As the fat layer of the anterior neck becomes thinner, the underlying fibers and orientation of the platysma muscle become visible through the skin. This is more likely to occur in patients with thin skin. When significant, this problem can be reduced by fat injections, although irregularities in the placement and resorption of fat can often create further irregularities and become a problem. It is important that great care be taken in revision surgery to treat visible banding in the neck. The dermis in such cases is often attached by scar tissue to the platysma muscle, and dissection can risk injury to a skin flap or to the platysma muscle itself, creating the possibility of buttonholing of the skin or of injury to the pseudo-marginal mandibular nerve. A cobra deformity can be created in several different ways. It is commonly the result of overly aggressive central or submental defatting of the 
neck either superficial to or deep to the platysma muscle. Defatting of the neck is most easily accomplished via a submental incision, with the submental region under direct visualization and vulnerable to overtreatment and the lateral neck not being visualized and being undertreated. This creates a classic cobra deformity with a depressed central region. A similar effect can be created by neglect undertreatment or by dehiscence of a ptotic platysma muscle. If the cause of a cobra defect is untreated lateral fat in the neck, its correction can be achieved by appropriate lateral defatting of the neck. This can also improve the appearance created by overly aggressive central defatting, but care must be taken to avoid muscle banding. A cobra defect caused by inadequate imbrication of the platysma muscle can be reversed by further imbrication of the muscle. A ridge can be created by an abrupt change in fat content in adjacent regions of the neck. This unnatural transition in fat content can be created at the inferior border of the neck by dissection in a heavy neck that requires significant defatting. Attention to detail will prevent this problem, and minor lipocontouring can usually remove persistent ridges.

\section{Other Medical Complications}

\section{Deep Venous Thrombosis and Venous Thromboembolism}

Deep venous thrombosis (DVT) and venous thromboembolism (VTE) are very rare complications of surgery of the neck and face. Hsu et $\mathrm{al}^{4}$ in a review, noted that DVT/VTE remains a small risk to patients undergoing elective plastic surgery. An exception to this is the increased risk of DVT/VTE with prolonged operating times in surgery of the face and neck, especially when done under general anesthesia, and in patients with multiple risk factors with surgery done under general anesthesia. Reinisch et $\mathrm{al}^{5}{ }^{5}$ in a study of DVT/VTE after facelift surgery, estimated a $0.35 \%$ incidence of DVT and a $0.14 \%$ incidence of VTE. Nearly all the patients who were found to experience DVT/VTE in this study were patients having surgery performed under general anesthesia. When general anesthesia was eliminated from the assessment, the adjusted risk decreased to $0.05 \%$ for DVT and $0.02 \%$ for VTE. This equates to a risk of VTE in 1 in 5,000 patients undergoing ambulatory facial cosmetic procedures performed under local anesthesia or with supplemental mild intravenous sedation. In addition to the greater risk created by general anesthesia and prolonged surgery done under general anesthesia, other risk factors for developing DVT in the short-term (30-day) postoperative period were examined in a prospective cohort of 21,903 consecutive surgical patients which concluded a history of myocardial infarction increased the risk of deep vein thrombosis cancer, increased the risk of all thromboembolism, and trend analysis showed that preexisting morbidities will increase by 1.5 -fold and thromboembolic events will increase by 3 -fold during the next decade. ${ }^{6}$

\section{Postoperative Depression}

Postoperative depression in patients undergoing plastic surgery of the face and neck is not uncommon. The initial shock of seeing the bruised and swollen surgical sites and the realization that the anticipated results of the surgery are not immediate contribute to this temporary emotional disturbance. It is also not uncommon in the early postoperative period for a patient to question the decision to have undergone a surgical procedure. The physician and staff should be aware of this and be prepared to provide the reassurance needed to support the patient until the patient can be excited about the results of an aesthetic surgical procedure. In many cases, preoperative patient education will help toward this and provide the surgeon and staff with the groundwork for reminding the patient that this sometimes occurs and that it will be short lived. Having the services of an aesthetician or someone to help apply makeup at approximately 10 days to 2 weeks postoperatively will also remind the patient that complete recovery is in sight. The surgeon and staff should encourage patients to begin resuming social activities at about this same time, because patients' apprehensions about how they will be perceived can fuel anxiety and mild depression.

\section{References}

1 Truswell WH. The facelift: a guide for safe, reliable, and reproducible results. In: Truswell WH, ed. Surgical Facial Rejuvenation: A Roadmap to Safe and Reliable Outcomes. New York, NY: Thieme Medical Publishers; 2009:24-46

2 Adamson PA, Moran ML. Complications of cervicofacial rhytidectomy. Facial Plast Surg Clin North Am 1993;1:257-271

3 Moyer JS, Baker SR. Complications of rhytidectomy. Facial Plast Surg Clin North Am 2005;13(03):469-478

4 Hsu P, Basu CB, Venturi M, Davison S. Venous thromboembolism prophylaxis. Semin Plast Surg 2006;20(04):225-231

5 Reinisch JF, Bresnick SD, Walker JWT, Rosso RF. Deep venous thrombosis and pulmonary embolus after face lift: a study of incidence and prophylaxis. Plast Reconstr Surg 2001;107(06): 1570-1575, discussion 1576-1577

6 Kikura M, Takada T, Sato S. Preexisting morbidity as an independent risk factor for perioperative acute thromboembolism syndrome. Archives of Surgery (Chicago, IL: 1960). 2006;140:1210-1217; discussion 1218 